Check for updates

Cite this: RSC Adv., 2018, 8, 6125

\title{
Effect and mechanism of oyster hydrolytic peptides on spatial learning and memory in mice
}

\author{
Xueqin Wang, (D) ${ }^{\text {ab }}$ Huahua Yu, ${ }^{\text {ab }}$ Ronge Xing, ${ }^{\text {ab }}$ Song Liu, ${ }^{\text {ab }}$ Xiaolin Chen ${ }^{\text {ab }}$ \\ and Pengcheng $\mathrm{Li}^{\star \mathrm{ab}}$
}

Oysters (Crassostrea talienwhanensis) contain large amounts of protein and exhibit many biological activities. This study was aimed at preparing oyster protein hydrolysates (OPH) and evaluating the $\mathrm{OPH}$ based on a spatial learning and memory capacity. A response surface methodology was employed to optimize hydrolysis conditions to determine the $\mathrm{OPH}$ with the highest AChE inhibitory activity, and the optimum extraction conditions were as follows: enzyme concentration of $1444.88 \mathrm{U} \mathrm{g}^{-1}, \mathrm{pH}$ of 7.38 , extraction temperature of $45{ }^{\circ} \mathrm{C}$, extraction time of $5.56 \mathrm{~h}$ and a water/material ratio of $2.45: 1$, and the minimum acetylcholinesterase (AChE) activity was $0.069 \mathrm{mM} \mathrm{min}{ }^{-1}$. The spatial memory and learning abilities and passive avoidance in mice were determined by using the Morris water maze test and a dark/ light avoidance test. Furthermore, the OPH group could relieve oxidative stress, reduce AChE levels, increase choline acetyltransferase (ChAT) levels and alleviate inflammatory reaction through reduction of interleukin-1 $\beta$ (IL-1 $\beta$ ), interleukin-6 (IL-6), and tumor necrosis factor- $\alpha$ (TNF- $\alpha$ ) levels. Additionally, upregulated expressions of brain-derived neurotrophic factor (BDNF) and neural cell adhesion molecules (NCAM) were observed in mice treated with $\mathrm{OPH}$. These findings suggested that $\mathrm{OPH}$ could be a functional food candidate to improve the learning and memory ability associated with oxidative stress and inflammatory reactions.

Received 8th December 2017 Accepted 25th January 2018

DOI: 10.1039/c7ra13139a

rsc.li/rsc-advances hepatotoxicity and excitotoxicity. ${ }^{8}$ Currently, the demand for finding alternative therapeutic agents to synthetic chemicals, such as natural products, is increasing because they are generally regarded as safer than their synthetic counterparts. ${ }^{9}$

Some researchers have found that the higher memory capacity a person has, the slower the decline in his or her cognitive ability during the early stage of $\mathrm{AD} \cdot{ }^{10}$ In recent years, researchers have focused on the impairment of learning and memory. Despite the research, the most effective approach and the precise underlying mechanism for memory-enhancing effects remained unknown. ${ }^{11}$ Growing evidences shows that oxidative stress resulting from reactive oxygen species (ROS) seriously affects $\mathrm{AD}$ animals and patients. ${ }^{12,13}$ Other hypothesis showed that inflammation clearly occurs in the AD-affected brain,${ }^{14}$ and neuroinflammation is now recognized as a prominent feature in the pathology of Alzheimer's, making it a potential target for disease therapy and prevention. ${ }^{15}$ Furthermore, the current strategy of AD intervention is mainly to ameliorate the cognitive symptoms related to acetylcholine (ACh) depletion, thereby enhancing the central cholinergic neurotransmission by reversing acetylcholinesterase (AChE) inhibition. ${ }^{16}$ Experimental studies suggest $\mathrm{ACh}$ plays a vital role in learning and memory. ${ }^{17}$ In addition, some researchers have studied interrelated physiological indicators in mice to evaluate the effect of products in improving the memory. Behavioral tests, such as the Morris water maze, dark/light avoidance and
${ }^{a}$ Key Laboratory of Experimental Marine Biology, Institute of Oceanology, Chinese Academy of Sciences, No. 7, Nanhai Road, Qingdao 266071, China. E-mail: pcli@ qdio.ac.cn; Fax: +86532 82968951; Tel: +86 53282898707

${ }^{b}$ Laboratory for Marine Drugs and Bioproducts of Qingdao National Laboratory for Marine Science and Technology, No. 1, Wenhai Road, Qingdao 266237, China 
other passive avoidance tests are used to evaluate the effects of treatment on learning and memory ability in animal models known to be sensitive to hippocampal-dependent learning and memory deficits. ${ }^{\mathbf{9}, 18}$

Oysters are excellent sources of high-quality nutrition in Northeast China and in other parts of the world. ${ }^{19}$ The oyster species, Crassostrea talienwhanensis, is rich in protein, low in fat and is a good source of several vitamins and minerals. $C$. talienwhanensis is used to study a variety of biological activities, including antioxidant activity, ${ }^{19}$ antihypertensive activity, ${ }^{20}$ antibacterial activity, ${ }^{21}$ antitumor activity ${ }^{22}$ and anti-inflammatory activity. ${ }^{23}$ Additionally, Lin et al. ${ }^{24}$ had researched the enzymatic hydrolysates from oyster protein on the ability of learning memory in mice, and found the hydrolysates had remarkable antioxidant activity in vivo, however, the mechanisms of the oyster protein hydrolysates (OPH) on the learning and memory capacity needed fully explored. With this idea in mind, we prepared OPH by using AChE activity in the astrocyte as a test model in vitro. The Morris water maze and dark/light avoidance tests, along with a series of biochemical indicators determinations were performed on mice in vivo to explore the relationship of spatial learning and memory mechanism with antioxidative stress and anti-inflammatory activity.

\section{Materials and methods}

\subsection{Materials}

Oyster (Crassostrea talienwhanensis) was purchased from a seafood market in Qingdao, China. Upon arrival, the oyster was washed, and the edible meat was separated from the shells, homogenized and stored at $-20{ }^{\circ} \mathrm{C}$ until use.

Five proteases (compound proteinase, trypsin, papain, neutrase and flavourzyme) were purchased from Beijing Solarbio Science \& Technology Co., Ltd (Beijing, China); 1,1-diphenyl-2picryhydrazyl (DPPH) and phenazine methosulfate (PMS) were purchased from Sigma Chemical Co. (St Louis, MO, USA); Nicotinamide-adenine dinucleotide (NADH) and nitroblue tetrazolium (NBT) were purchased from Ruitaibio Co (Beijing, China). The Medical College of Qingdao University (Qingdao, China) provided astrocytes. The growth medium and antibiotics for the cell culture experiments were purchased from Hyclone (USA). Fetal bovine serum was purchased from Gibco (Australia). All other chemicals and solvents were of analytical grade.

\subsection{Methods}

2.2.1 Preparation of OPH. Hydrolysis was performed by first mixing oyster homogenate with deionized water at a ratio of $1: 5(\mathrm{w} / \mathrm{v})$. The mixtures were adjusted to the required $\mathrm{pH}$ with $0.01 \mathrm{~mol} \mathrm{~L}^{-1} \mathrm{NaOH}$ or $\mathrm{HCl}$, and heated in a water bath to the required temperature before the five proteases were added in proper proportion based on activity. Next, the hydrolysis reactions were carried out in a shaking incubator. At the end of the hydrolysis period, the mixtures were heated in boiling water for 10 minutes to inactivate the protease. Following inactivation, the hydrolysates were centrifuged at $10000 \times g\left(4{ }^{\circ} \mathrm{C}\right)$ for 15 minutes, and the supernatants were spray-dried to obtain
$\mathrm{OPH}$, which was saved for further study. Among the five hydrolysates, the one with the highest antioxidant activity and AChE inhibition rate was chosen for further optimization according to single factor experiment and response surface methodology (RSM).

2.2.2 AChE activity assays. As the most widely distributed type of cell in the mammalian brain, astrocytes are important players in neurological disorders and participate and modulate neuronal communication. AChE has critical catalytic function in cholinergic synapses and is also present in astrocytes. In this study, astrocytes were cultured in DMEM/F12 medium containing $10 \%$ fetal bovine serum and 1\% antibiotic and antimycotic solution, and incubated. The medium was changed every two days.

Astrocytes were seeded in 24-well plates at a final concentration of $1 \times 10^{5}$ to $10^{6}$ cells per $\mathrm{mL}$, and $0.5 \mathrm{~mL} \mathrm{OPH}$ (10 $\mathrm{mg} \mathrm{mL}{ }^{-1}$ ) was added for 24 hours. After the removal of culture medium, $0.5 \mathrm{~mL}$ phosphate buffer $(0.01 \mathrm{M}, \mathrm{pH} 7.2)$ was added in 24-well plates and the astrocytes were ground with a grinding rod.

Finally, the phosphate buffer was centrifuged at $5000 \times g$ $\left(4{ }^{\circ} \mathrm{C}\right)$ for 10 minutes, and the supernatant was used for testing AChE activity using an AChE kit.

$$
\begin{aligned}
& \text { AChE activity }\left(\mathrm{mM} \min ^{-1}\right)=\frac{A_{\text {sample }}-A_{\text {control }}}{A_{\text {standard }}-A_{\text {blank }}} \\
& \times \text { standard concentration }\left(\mu \mathrm{mol} \mathrm{mL}^{-1}\right) / \text { reaction time }(\min )
\end{aligned}
$$

2.2.3 Determination of soluble peptide content. The content of soluble peptides was determined by the biuret method according to our previous report. ${ }^{25}$ To complete this method, $4.0 \mathrm{~mL}$ biuret reagent was added to a $1.0 \mathrm{~mL}$ sample. The mixture was shaken and left to stand for 30 minutes, and then the absorbance was measured at $540 \mathrm{~nm}$. The biuret reagent was used as the control and BSA was used as the standards. All experiments were performed in triplicate.

2.2.4 Scavenging activity on hydroxyl radical. Scavenging activity of $\mathrm{OPH}$ on hydroxyl radicals was performed, using method described by Smeriglio et al. ${ }^{26}$ with a few modifications. Briefly, the reaction mixture contained $1.0 \mathrm{~mL}$ of phosphate buffer (PBS, $0.15 \mathrm{~mol} \mathrm{~L}^{-1}, \mathrm{pH} 7.4$ ), $1.0 \mathrm{~mL}$ of safranine $\mathrm{T}$ $(1.0 \mathrm{mM}), 0.5 \mathrm{~mL}$ of EDTA-FeSO ${ }_{4}\left(2.0 \mathrm{mmol} \mathrm{L}^{-1}\right)$ and $1.0 \mathrm{~mL}$ of $\mathrm{OPH}$ with the concentration of $5 \mathrm{mg} \mathrm{mL}^{-1}$, the same concentration of vitamin $\mathrm{C}(\mathrm{Vc})$ was used as standard. After sufficient mixing, $1.0 \mathrm{~mL}$ of $\mathrm{H}_{2} \mathrm{O}_{2}(3 \%)$ was added to the mixture. Following incubation at $37{ }^{\circ} \mathrm{C}$ for $30 \mathrm{~min}$, the absorbance of the mixture was measured at $520 \mathrm{~nm}$. The hydroxyl radical scavenging activity was calculated as: scavenging rate $(\%)=$ $\left[\left(A_{1}-A_{0}\right) /\left(A_{2}-A_{0}\right)\right] \times 100$, where $A_{1}$ was the absorbance of OPH, $A_{2}$ was the absorbance without $\mathrm{H}_{2} \mathrm{O}_{2}, A_{0}$ was the absorbance of the control. Both $A_{0}$ and $A_{2}$ were the mixtures with sample solution replaced by deionized water. All experiments were performed in triplicate.

2.2.5 Scavenging activity on DPPH radical. The DPPH radical scavenging activity of the $\mathrm{OPH}$ was determined as described by Blois ${ }^{27}$ with slight modifications. Briefly, $1.0 \mathrm{~mL}$ of DPPH $\left(0.1 \mathrm{mmol} \mathrm{L}^{-1}\right)$ diluted in ethanol was added to $3.0 \mathrm{~mL}$ of 
$\mathrm{OPH}$ with the concentration of $5 \mathrm{mg} \mathrm{mL}^{-1}$, the same concentration of Vc was used as standard. After vigorous shaking, the mixture was left to stand for $30 \mathrm{~min}$ and the absorbance was measured at $517 \mathrm{~nm}$. The DPPH radical scavenging activity was calculated as follows: scavenging rate $(\%)=\left[1-\left(A_{1}-A_{0}\right) /\left(A_{2}-\right.\right.$ $\left.\left.A_{0}\right)\right] \times 100$, where $A_{0}$ was the absorbance without DPPH, $A_{1}$ was the absorbance in the presence of the $\mathrm{OPH}$, and $A_{2}$ was the absorbance of the control (without sample). All experiments were performed in triplicate.

2.2.6 Scavenging activity on superoxide radical. The superoxide radical scavenging activity of $\mathrm{OPH}$ was assessed based on the method of Nishikimi et al. ${ }^{28}$ with a slight modification. The reaction mixture, containing $\mathrm{OPH}$ with the final concentration of $5 \mathrm{mg} \mathrm{mL}^{-1}, 0.5 \mathrm{~mL}$ PMS $\left(20 \mathrm{~mol} \mathrm{~L}^{-1}\right), 0.5 \mathrm{~mL}$ NADH $\left(240 \mathrm{~mol} \mathrm{~L}^{-1}\right)$ and $0.5 \mathrm{~mL} \mathrm{NBT}\left(150 \mathrm{~mol} \mathrm{~L}^{-1}\right)$ in $3.0 \mathrm{~mL}$ Tris-HCl buffer $\left(0.1 \mathrm{~mol} \mathrm{~L}^{-1}, \mathrm{pH} 7.4\right)$, was incubated at room temperature for $5 \mathrm{~min}$ and the absorbance was read at $560 \mathrm{~nm}$ against a blank. The same concentration of Vc was used as standard. The capability of scavenging to superoxide radical was calculated using the following equation: scavenging effect $(\%)=$ $\left[1-\left(A_{1} / A_{0}\right)\right] \times 100$, where $A_{1}$ was the absorbance in the presence of the sample, and $A_{0}$ was the absorbance of the control. All experiments were carried out in triplicate.

2.2.7 Animal test. One hundred and twenty Kunming male mice (18-22 g, approval no. SCXK 2012-003) were provided by the Experimental Animal Center of Xi'an Jiaotong University Health Science Center. Four-week old mice were allowed to adapt to their surroundings for one week before starting the experiments, and were housed in stainless steel cages (ten mice per cage) at room temperature $\left(20-26{ }^{\circ} \mathrm{C}\right)$, with humidity ranging from 40 to $60 \%$, and a $12 / 12$ hour light-dark cycle. The mice had free access to water and a standard rodent diet. After adaptation, the mice were divided into two parts, each part contained five groups, and there were twelve mice in each group: (1) normal control (NC) group, (2) positive control (PC) group, (3) low-dose OPH (OPH-L) group, (4) middle-dose $\mathrm{OPH}$ (OPH-M) group, (5) high-dose OPH (OPH-H) group. The NC group was gavaged $0.4 \mathrm{~mL}$ normal saline. The PC group was gavaged 0.4 mL huperzine water solution (Shanghai Fudan Fuhua Pharmaceutical Co., Ltd, Shanghai, China) at a dose of $0.05 \mathrm{mg} \mathrm{kg}^{-1}$. The OPH groups were gavaged $0.4 \mathrm{~mL} \mathrm{OPH}$ water solution with the dose of $150 \mathrm{mg} \mathrm{kg} \mathrm{kg}^{-1}, 500 \mathrm{mg} \mathrm{kg}^{-1}$ and $1500 \mathrm{mg} \mathrm{kg}^{-1}$, respectively. One part mice were subject to the Morris water maze test and the other part mice were subject to the dark/light avoidance test at the end of the treatment period.

2.2.8 Morris water maze test. Spatial learning and memory ability of the mice was evaluated by the Morris water maze test, which has become one of the most frequently used laboratory tools in behavioral neuroscience. ${ }^{\mathbf{2 9 , 3 0}}$ The experimental apparatus, consisting of a blank circular tank (diameter $120 \mathrm{~cm}$, height $50 \mathrm{~cm})$, filled with water $\left(24 \pm 1{ }^{\circ} \mathrm{C}\right)$ to a height of $30 \mathrm{~cm}$, and was located in a test room. The tank was divided into four equal quadrants, and a movable circular platform $(9 \mathrm{~cm}$ diameter) was placed in the fourth quadrant, submerged $1 \mathrm{~cm}$ below the water surface, and invisible. After 30 days of oral administration, the mice were placed in water to swim freely for two minutes to become familiar with the environment on the first day. A trial began by releasing the mice into the water pool from one of the four different starting positions facing towards the wall of the maze. The mice were trained to swim to the platform in the circular tank. If a mouse was unable to find the platform within 90 seconds, it was guided to the platform and placed on the platform for 10 seconds. The four quadrants training are completed as one trial. The mice received three training trials per day for five consecutive days, and escape latency was measured as a learning score by an auto-tracking system.

A spatial probe trial was carried out after five days. In this test, the platform was removed from the water tank. The mice were placed into the water pool from one of the four different starting positions facing towards the wall of the maze, and total swimming distance, swimming time and frequency of going into the quadrants of the mice in the trail were recorded. Based on the above experiment data, the percentage of swimming distance/time in the quadrant to the total distance/time would reflect the spatial memory ability of the mice on the location of the platform.

2.2.9 Dark/light avoidance test. After 45 days of oral administration, the dark/light avoidance test was carried out from day 46 to day 50, according to Yamaguchi et al. ${ }^{31}$ with some modification. The apparatus consisted of light $(12 \mathrm{~cm} \times 4.5 \mathrm{~cm})$ and dark $(17 \mathrm{~cm} \times 4.5 \mathrm{~cm})$ chambers; a door with a diameter of $3 \mathrm{~cm}$ was set for separation of the two chambers. Electric wires, arranged with a parallel interval of $1 \mathrm{~cm}$, were set in the two chambers, and delivered an electric shock (0.3-0.4 $\mathrm{mA})$ in the dark chamber. In each trial, a mouse was placed into the light chamber first with the shuttle door open. After the mouse entered the dark chamber, an escape failure was recorded and an inescapable electric shock was delivered through the electric wires. The mouse was removed from the dark chamber and the trial ended. The trial also ended if the mouse remained in the light chamber for 300 seconds. The avoidance test was repeated after 24 hours, and the memory reappearance experiment was carried out after five days, which could estimate the ability of memory in mice.

2.2.10 Biochemical parameters of mice. After completing the behavioral studies, the mice were anesthetized and decapitated, and the blood was collected. The brain, spleen and thymus were taken out and weighed. The hippocampus was separated from the whole brain on ice, blotted gently with filter paper to remove blood and extraneous tissue fragments, then flash frozen with liquid nitrogen and stored at $-80^{\circ} \mathrm{C}$ until use. The hippocampus was suspended in ice-cold, normal saline at a ratio of $1: 9$. The homogenate was centrifuged at $10000 \times g$ for 30 minutes, and the supernatant was used for a biochemical assay.

The contents of ROS, malondialdehyde (MDA), superoxide dismutase (SOD), glutathione peroxidase (GSH-Px), tumor necrosis factor- $\alpha$ (TNF- $\alpha$ ), interleukin-6 (IL-6), interleukin-1 $\beta$ (IL-1 $\beta$ ), AChE and choline acetyltransferase (ChAT) were determined using assay kits (Institute of Biological Engineering of Nanjing Jiancheng, Nanjing, China). Brain-derived neurotrophic factor (BDNF) and neural cell adhesion molecules (NCAM) were determined using ELISA testing (Institute of Biological Engineering of Nanjing Jiancheng, Nanjing, China). In 
addition, the levels of BDNF and NCAM protein expressions were determined by western blot. This section was conducted by Xi'an United Nations Quality Detection Technology CO., Ltd (Xi'an, China).

\subsection{Statistical analysis}

Data were presented as means $\pm \mathrm{SD}$. The statistical significance of the data was determined by variance analysis (ANOVA) using the SPSS software (version 18.0 for Windows, SPSS Inc., Chicago, IL, USA) and means were compared by Duncan's multiple comparison post-test. Statistical differences were considered to be significant at $p<0.05$.

\section{Results and discussions}

\subsection{Selections of proteolytic enzymes}

The types of enzymes that affect antioxidant activity and other biological activities of enzymatic hydrolysis ${ }^{32}$ is critical to select an appropriate protease because this leads to different peptide bond cleavage patterns in protein hydrolysates according to the enzyme used. ${ }^{33}$ In this study, five proteases were used for hydrolytic production of OPH: protamex, trypsin, papain, neutrase and flavourzyme. Selection of these proteases was based on the ability of each enzyme to reach high radical scavenging activities and low AChE activity. As shown in Table 1, hydrolysate treated with protamex demonstrated the highest soluble peptide content of $5.86 \mathrm{~g} / 100 \mathrm{~mL}$, hydroxyl radical scavenging activity of $70.78 \%$, DPPH radical scavenging activity of $83.41 \%$ and superoxide radical scavenging activity of $62.53 \%$, which were significantly different from other four proteases hydrolysates $(P<0.05)$, and protamex hydrolysate also showed considerable radical scavenging activities compared to the standard antioxidant (Vc). These results were consistent with those reported by Jia et al., ${ }^{34}$ where Alaska pollack skin was treated with six kinds of proteases, showing protamex hydrolysate exhibiting the highest yield of peptide and antioxidant activities. Furthermore, the catalytic activity of AChE was confirmed through complete inhibition with AChE inhibitor. ${ }^{35}$ The protamex hydrolysate exhibited the lowest AChE activity of $0.080 \mathrm{mM} \min ^{-1}$, meaning that $\mathrm{OPH}$ prepared with protamex could better inhibit AChE activity. Therefore, protamex was chosen as the best candidate for further study.

\subsection{Single factor experiments}

In this work, the effects of five single factors on the AChE activity were investigated. Results showed that under the range of these five single factors, the AChE activity decreased at first followed by an increase. As such, optimal conditions were determined as follows: enzyme concentration of $1200 \mathrm{U} \mathrm{g}^{-1}$, extraction time of 5.0 hours, $\mathrm{pH}$ of 8.0 , a water/material ratio of $2: 1$ and extraction temperature of $55^{\circ} \mathrm{C}$.

\subsection{Optimization of extraction conditions by BBD}

The single factor experiments involved five independent variables at five levels employed in a Box-Behnken Design (BBD). The five independent variables, enzyme concentration, extraction time, $\mathrm{pH}$, water/material ratio and extraction temperature, were coded as $X_{1}, X_{2}, X_{3}, X_{4}$ and $X_{5}$, respectively. The ranges and levels of the variables were given by the Design Expert 8.0 (StatEase. Inc., China), which was used to analyze and calculate the predicted responses and experimental design for the AChE activity. The design matrix and corresponding results obtained from BBD for determining the effects of the five independent variables, $X_{1}, X_{2}, X_{3}, X_{4}$ and $X_{5}$, were listed in Table 2 .

These results showed that the AChE activity ranged from

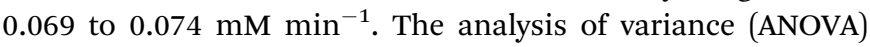
results for the model were given in Table 3 . The corresponding variables were more significant as the $F$-value became greater and the $P$-value became smaller. ${ }^{36}$ The model $F$-value of 11.99 implies the model was significant. The value of "Prob $>F$ " of less than 0.0500 indicated that the model terms were significant. Additionally, it could be stated that the variables with significant effects on the AChE activity of OPH were linear terms $\left(X_{1}, X_{2}, X_{4}\right.$ and $\left.X_{5}\right)$, quadratic terms $\left(X_{1}{ }^{2}, X_{2}{ }^{2}, X_{3}{ }^{2}\right.$ and $\left.X_{4}{ }^{2}\right)$. Furthermore, the model showed good fit with the experimental data, with high values of $R^{2}(90.56 \%)$ and adj. $R^{2}(83.00 \%)$. The low coefficient value of the variation $(\mathrm{CV}=0.66 \%)$ clearly suggested a high degree of precision and reliability of the experimental values. This result implied that the hydrolysis process of OPH could be analyzed and predicted by the model.

Using Design-Expert 8.0, the optimal hydrolysate conditions were as follows: enzyme concentration of $1444.88 \mathrm{U} \mathrm{g}^{-1}$, extraction time of 5.56 hours, $\mathrm{pH}$ of 7.38 , and a water/material ratio of $2.45: 1$ and extraction temperature of $45{ }^{\circ} \mathrm{C}$. The minimum AChE activity was $0.069 \mathrm{mM} \mathrm{min}^{-1}$, which was in

Table 1 The soluble peptide content, radical scavenging activities and AChE activity of OPH prepared by different proteases ${ }^{a}$

\begin{tabular}{llllll}
\hline Protease & $\begin{array}{l}\text { Soluble peptide content } \\
(\mathrm{g} / 100 \mathrm{~mL})\end{array}$ & $\begin{array}{l}\text { Hydroxyl radical scavenge } \\
\text { activity (\%) }\end{array}$ & $\begin{array}{l}\text { DPPH radical scavenging } \\
\text { activity (\%) }\end{array}$ & $\begin{array}{l}\text { Superoxide-radical } \\
\text { scavenging }(\%)\end{array}$ & $\begin{array}{l}\text { AChE activity }\left(\mathrm{U} \mathrm{mL}^{-1}\right) \\
\text { Achen }\end{array}$ \\
\hline Protamex & $5.86 \pm 0.04^{\mathrm{a}}$ & $70.78 \pm 0.30^{\mathrm{a}}$ & $83.41 \pm 0.92^{\mathrm{a}}$ & $62.53 \pm 0.48^{\mathrm{a}}$ & $0.080 \pm 0.01^{\mathrm{c}}$ \\
Trypsin & $4.26 \pm 0.01^{\mathrm{b}}$ & $64.93 \pm 0.70^{\mathrm{b}}$ & $77.42 \pm 0.46^{\mathrm{c}}$ & $46.83 \pm 0.28^{\mathrm{c}}$ & $0.086 \pm 0.01^{\mathrm{b}}$ \\
Papain & $4.04 \pm 0.12^{\mathrm{c}}$ & $60.89 \pm 1.08^{\mathrm{e}}$ & $77.11 \pm 0.70^{\mathrm{c}}$ & $31.04 \pm 1.88^{\mathrm{e}}$ & $0.085 \pm 0.01^{\mathrm{b}}$ \\
Flavourzyme & $2.42 \pm 0.06^{\mathrm{d}}$ & $62.87 \pm 0.93^{\mathrm{cd}}$ & $80.03 \pm 0.96^{\mathrm{b}}$ & $40.13 \pm 0.69^{\mathrm{d}}$ & $0.085 \pm 0.01^{\mathrm{b}}$ \\
Neutrase & $4.25 \pm 0.09^{\mathrm{b}}$ & $64.34 \pm 0.80^{\mathrm{bc}}$ & $68.97 \pm 0.96^{\mathrm{e}}$ & $25.34 \pm 1.65^{\mathrm{f}}$ & $0.088 \pm 0.01^{\mathrm{a}}$ \\
Vc & & $62.46 \pm 1.05^{\mathrm{d}}$ & $71.88 \pm 0.67^{\mathrm{d}}$ & $55.09 \pm 0.61^{\mathrm{b}}$ &
\end{tabular}

${ }^{a}$ Data were presented as means \pm SD. Values followed by different superscript in the same columns were significantly different $(P<0.05)$ according to variance analysis (ANOVA) using the SPSS software and means were compared by Duncan's multiple comparison post-test. Statistical differences were considered to be significant at $p<0.05$. 
Table 2 Experimental design and result of response surface

\begin{tabular}{|c|c|c|c|c|c|c|}
\hline Run numbers & $X_{1}$ & $X_{2}$ & $X_{3}$ & $X_{4}$ & $X_{5}$ & $\begin{array}{l}\text { AChE activity } \\
\left(\mathrm{mM} \mathrm{min}{ }^{-1}\right)\end{array}$ \\
\hline 1 & 800 & 3 & 8 & 2 & 55 & 0.074 \\
\hline 2 & 1600 & 3 & 8 & 2 & 55 & 0.072 \\
\hline 3 & 800 & 7 & 8 & 2 & 55 & 0.072 \\
\hline 4 & 1600 & 7 & 8 & 2 & 55 & 0.070 \\
\hline 5 & 1200 & 5 & 7 & 1 & 55 & 0.071 \\
\hline 6 & 1200 & 5 & 9 & 1 & 55 & 0.072 \\
\hline 7 & 1200 & 5 & 7 & 3 & 55 & 0.070 \\
\hline 8 & 1200 & 5 & 9 & 3 & 55 & 0.070 \\
\hline 9 & 1200 & 3 & 8 & 2 & 45 & 0.072 \\
\hline 10 & 1200 & 7 & 8 & 2 & 45 & 0.070 \\
\hline 11 & 1200 & 3 & 8 & 2 & 65 & 0.073 \\
\hline 12 & 1200 & 7 & 8 & 2 & 65 & 0.071 \\
\hline 13 & 800 & 5 & 7 & 2 & 55 & 0.072 \\
\hline 14 & 1600 & 5 & 7 & 2 & 55 & 0.070 \\
\hline 15 & 800 & 5 & 9 & 2 & 55 & 0.072 \\
\hline 16 & 1600 & 5 & 9 & 2 & 55 & 0.070 \\
\hline 17 & 1200 & 5 & 8 & 1 & 45 & 0.070 \\
\hline 18 & 1200 & 5 & 8 & 3 & 45 & 0.069 \\
\hline 19 & 1200 & 5 & 8 & 1 & 65 & 0.072 \\
\hline 20 & 1200 & 5 & 8 & 3 & 65 & 0.070 \\
\hline 21 & 1200 & 3 & 7 & 2 & 55 & 0.072 \\
\hline 22 & 1200 & 7 & 7 & 2 & 55 & 0.070 \\
\hline 23 & 1200 & 3 & 9 & 2 & 55 & 0.072 \\
\hline 24 & 1200 & 7 & 9 & 2 & 55 & 0.071 \\
\hline 25 & 800 & 5 & 8 & 1 & 55 & 0.072 \\
\hline 26 & 1600 & 5 & 8 & 1 & 55 & 0.071 \\
\hline 27 & 800 & 5 & 8 & 3 & 55 & 0.071 \\
\hline 28 & 1600 & 5 & 8 & 3 & 55 & 0.070 \\
\hline 29 & 1200 & 5 & 7 & 2 & 45 & 0.070 \\
\hline 30 & 1200 & 5 & 9 & 2 & 45 & 0.070 \\
\hline 31 & 1200 & 5 & 7 & 2 & 65 & 0.070 \\
\hline 32 & 1200 & 5 & 9 & 2 & 65 & 0.070 \\
\hline 33 & 800 & 5 & 8 & 2 & 45 & 0.071 \\
\hline 34 & 1600 & 5 & 8 & 2 & 45 & 0.069 \\
\hline 35 & 800 & 5 & 8 & 2 & 65 & 0.072 \\
\hline 36 & 1600 & 5 & 8 & 2 & 65 & 0.071 \\
\hline 37 & 1200 & 3 & 8 & 1 & 55 & 0.072 \\
\hline 38 & 1200 & 7 & 8 & 1 & 55 & 0.071 \\
\hline 39 & 1200 & 3 & 8 & 3 & 55 & 0.072 \\
\hline 40 & 1200 & 7 & 8 & 3 & 55 & 0.070 \\
\hline 41 & 1200 & 5 & 8 & 2 & 55 & 0.070 \\
\hline 42 & 1200 & 5 & 8 & 2 & 55 & 0.070 \\
\hline 43 & 1200 & 5 & 8 & 2 & 55 & 0.069 \\
\hline 44 & 1200 & 5 & 8 & 2 & 55 & 0.069 \\
\hline 45 & 1200 & 5 & 8 & 2 & 55 & 0.070 \\
\hline 46 & 1200 & 5 & 8 & 2 & 55 & 0.070 \\
\hline
\end{tabular}

agreement with the experimental value $\left(0.071 \mathrm{mM} \mathrm{min}^{-1}\right)$, suggesting a good fit between the model and experimental data.

\subsection{Effect of OPH on the body weight and viscera indexes of mice}

After 45 days of oral administration, the mice showed no mortality or behavioral abnormalities. In addition, body weight and viscera indexes of the mice were shown in Table 4. No differences in body weight were shown between the five treatment groups at the end of 45 days of normal saline, huperzine, OPH-L, OPH-M or OPH-H oral administration. In addition, there were no significant changes in brain and spleen indexes
Table 3 ANOVA for response surface quadratic model

\begin{tabular}{llllll}
\hline & $\begin{array}{l}\text { Sum of } \\
\text { squares }\end{array}$ & df $\begin{array}{l}\text { Mean } \\
\text { square }\end{array}$ & $F$ value & $\begin{array}{l}P \text { value } \\
\text { prob }>F\end{array}$ \\
\hline Model & $5.19 \times 10^{-5}$ & 20 & $2.59 \times 10^{-6}$ & 11.99 & $<0.0001$ \\
Residual & $5.41 \times 10^{-6}$ & 25 & $2.16 \times 10^{-7}$ & & \\
Lack of fit & $4.53 \times 10^{-6}$ & 20 & $2.27 \times 10^{-7}$ & 1.30 & 0.4180 \\
Pure error & $8.73 \times 10^{-7}$ & 5 & $1.75 \times 10^{-7}$ & & \\
Cor. total & $5.73 \times 10^{-5}$ & 45 & & & \\
$R^{2}$ & 0.9056 & & & & \\
Adj. $R^{2}$ & 0.8300 & & & & \\
Pred. $R^{2}$ & 0.6613 & & & & \\
Adeq. precision & 13.421 & 0.66 & & & \\
CV\% & & & & & \\
\end{tabular}

among the five groups of mice $(P<0.05)$. Only the thymus index was significant higher in the OPH-L group compared to the NC group. The result showed that the PC and OPH groups had no significant effect on the body weight and viscera indexes of mice.

\subsection{Latency of mice in Morris water maze}

We conducted a Morris water maze test to assess memory and learning ability of the mice treated with $\mathrm{OPH}$. Reference memory outcomes from Morris water maze test were shown in Fig. 1. After five days of training, escape latency in the five groups reduced, which proving the mice exhibited spatial learning and memory. On the first day, the escape latency of the NC group and PC group were about 44.40 seconds and 41.03 seconds, and the mice in the OPH groups showed better performance with the escape latency were 39.75 seconds, 41.10 seconds and 33.39 seconds respectively. During the five days of training, OPH administrations also resulted in shorter escape latency compared with the NC and PC groups. In addition, changes in path length produced by training trials in each group showed a pattern similar to that of escape latency.

Following the reference memory task, a spatial probe trial was carried out after five days. As shown in Fig. 2A, the OPH groups significantly shorten the total swimming distance, compared to the NC group $(P<0.05)$. Besides, OPH-M group showed the shortest total swimming distance by $11416.52 \pm$ $2491 \mathrm{~mm}$, which was also significantly shorter than that in the PC group $(P<0.05)$. Fig. 2B indicated that there was a significant group effect on the time spent in the quadrant, in which the platform was located at the same place during training (target quadrant). The NC group spent less time in the target quadrant than the OPH-M group $(p<0.05)$, moreover, compared to the PC group, $\mathrm{OPH}$ groups could also prolong the time that the mice spent in the target quadrant $(p<0.05)$, which showed the mice were consciously looking for the platform. We suggested that the performance of mice in $\mathrm{OPH}$ group was attributable to the effect of OPH. Additionally, swimming pathway is helpful in understanding the truth of the memory and learning ability of mice in spatial probe trial. Fig. 2C clearly indicated the swimming pathway in the five groups, the mice of $\mathrm{NC}$ group searched the target quadrant with directionless and disorder path; while 
Table 4 Effect of $\mathrm{OPH}$ on the body weight and viscera indexes of mice ${ }^{a}$

\begin{tabular}{|c|c|c|c|c|c|}
\hline \multirow[b]{2}{*}{ Groups } & \multicolumn{2}{|c|}{ Body weight $(\mathrm{g})$} & \multicolumn{3}{|c|}{ Viscera indexes $^{b}\left(\mathrm{mg} \mathrm{g}^{-1}\right)$} \\
\hline & Beginning & Ending & Brain & Spleen & Thymus \\
\hline $\mathrm{PC}$ & $19.83 \pm 2.02$ & $32.46 \pm 2.40$ & $13.28 \pm 1.23$ & $2.23 \pm 0.48$ & $2.98 \pm 0.69^{\mathrm{ab}}$ \\
\hline MPH-L & $20.33 \pm 1.56$ & $32.89 \pm 2.65$ & $12.65 \pm 1.27$ & $2.51 \pm 0.51$ & $2.71 \pm 0.49^{\mathrm{b}}$ \\
\hline МРH-M & $20.33 \pm 1.83$ & $31.33 \pm 3.01$ & $13.62 \pm 1.82$ & $2.36 \pm 0.61$ & $2.65 \pm 0.68^{b}$ \\
\hline
\end{tabular}

${ }^{a}$ Data were presented as means \pm SD. Mean values within the same column with different superscript were significantly different according to variance analysis (ANOVA) using the SPSS software and means were compared by Duncan's multiple comparison post-test. Statistical differences were considered to be significant at $p<0.05 .{ }^{b}$ Viscera indexes $=$ weight of brain, spleen or thymus $(\mathrm{mg}) / \mathrm{weight}$ of mice $(\mathrm{g}) \times 10$.

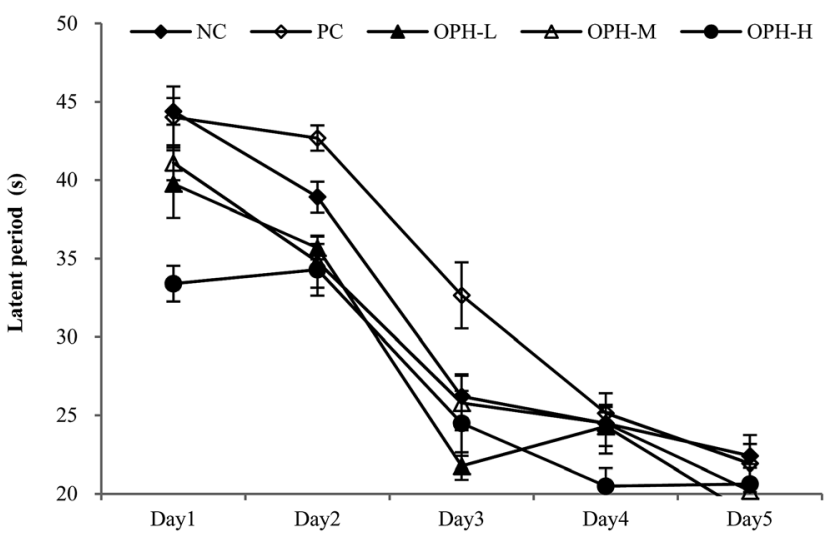

Fig. 1 Effect of OPH on performance in the training trials of reference memory task. After daily oral administration with normal saline (NC group), huperzine (PC group) or OPH (OPH-L, OPH-M and $\mathrm{OPH}-\mathrm{H}$ groups) for 30 days, animals were test in Morris water maze task. Values were presented as means \pm SD.

the $\mathrm{OPH}$ and PC groups showed the simple and clear path, which indicated the memory and learning ability of mice was improved.

\subsection{Dark/light avoidance test}

A dark/light avoidance test was used to evaluate the memory and learning ability in an animal model. ${ }^{11}$ Step-through latency from a light chamber to a dark chamber was used as a marker to evaluate memory and learning ability. ${ }^{29}$ As shown in Fig. 3, all the mice went into the dark chamber in a relatively short time because of skototaxis, with no significant difference in the five groups in the first trials. The aim of this test was to prove the mice went into the dark chamber and received an electric shock, whether they could remember the electric shock and avoided entering the dark chamber the next time.

Interestingly, when we placed the mice in the light chamber after 24 hours, the step-through latency of the five groups showed different behaviors in the second trial. The PC group showed the longest step-through latency by 257.3 seconds, which was significantly different from the NC, OPH-M and OPH$\mathrm{H}$ groups $(P<0.05)$. The result indicated that the mice in the PC group remembered the electric shock and stayed in the light chamber for the longest time. In addition, the OPH-L group stayed in the light chamber for a longer time compared to the NC group. In general dose-response model, an effect increases with dose increases. ${ }^{37}$ However, an interesting phenomenon to note was that the OPH groups exhibited no dose-dependence in the dark/light avoidance test.

The memory reappearance trial was carried out five days later, with the purpose of estimating the mice ability of memory. As shown in Fig. 3, the mice in NC group still spent the shortest time staying in the light chamber and the treatment with $\mathrm{OPH}$ significantly prolonged the latency of entering the dark chamber comparing with the NC group $(P<0.05)$. Interestingly, there was no significant difference between the $\mathrm{OPH}$ treated groups, which was similar to the result from Chai et al. ${ }^{9}$ In addition, the latency to enter the dark chamber was also longer in the PC group than those of the NC group.

\subsection{Effect of OPH relieved the oxidative stress in vivo}

In recent years, some studies revealed that antioxidative peptides could improve learning and memory ability via antioxidative stress. ${ }^{11}$ It is possible that antioxidative peptides with strong free radical-scavenging activity might exhibit some beneficial effect on impairment-related memory degeneration. ${ }^{11,38}$ It is certain now that oxidative stress can lead to cell and tissue damage, thus resulting in age-related cognitive decline. ${ }^{39}$ As such, it is reasonable to study the relationship between $\mathrm{OPH}$ and oxidative stress.

Oxidative stress refers to an imbalance between cellular productions of ROS and antioxidant enzymes, such as SOD and GSH-Px. ${ }^{40}$ ROS is a collective term for oxygen-centered radicals. In humans, uncontrolled generation of ROS can result in oxidative damage of cellular DNA, protein and lipids; even the development of Alzheimer's and inflammatory diseases. ${ }^{41,42}$ As shown in Fig. 4, the increase of ROS in the NC group reflected free radical accumulation after the dark/light avoidance test, this was consistent with those reported by Vollaard et al. ${ }^{43}$ who considered that exercise was accompanied by an increased generation of free radicals. While the ROS levels in OPH groups were significantly lower than that of the NC group $(P<0.05)$, and the results indicate that the $\mathrm{OPH}$ groups could eliminate free radicals and reduce oxidative stress. Moreover, the ROS level of the PC group was also significantly lower than that of the NC group. 

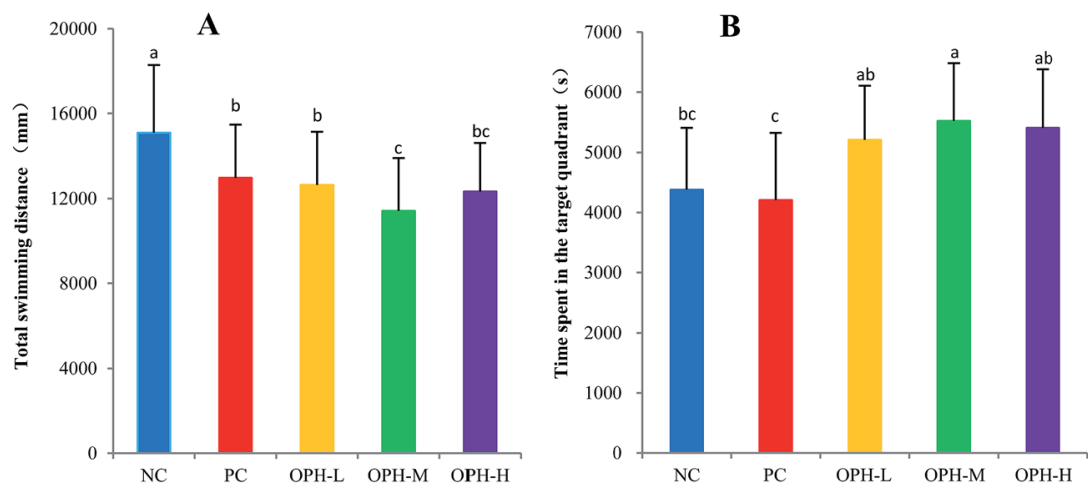

C

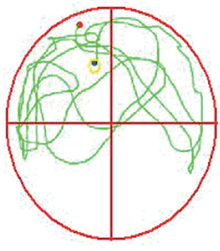

NC

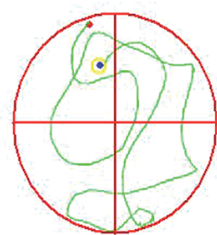

$\mathrm{PC}$

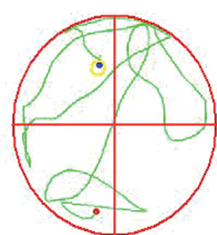

$\mathrm{OPH}-$

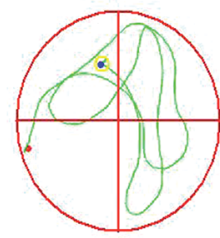

$\mathrm{OPH}-\mathrm{MI}$

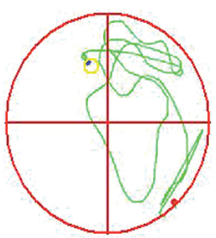

$\mathrm{OPH}-\mathrm{H}$

Fig. 2 Effect of $\mathrm{OPH}$ on performance in the probe trial of the water maze task. (A) Comparison of total swimming distance of each group. (B) The time of each group in the target quadrant in the probe trial. (C) The swimming pathway of each group in the probe trial. Data were presented as means \pm SD. Bar graphs followed by different letters were significantly different according to variance analysis (ANOVA) using the SPSS software and means were compared by Duncan's multiple comparison post-test. Statistical differences were considered to be significant at $p<0.05$.

The whole brain is vulnerable to free radical-induced damage because of its high oxygen consumption, abundant lipid content and relative paucity of antioxidant enzymes. ${ }^{\mathbf{4 4}}$ SOD and GSH-Px were actual indicators of the antioxidant capacity of the body and were involved in protection against damage caused by oxidative stress. Fig. 4 showed that the level of SOD was significantly increased in the OPH-H group compared with

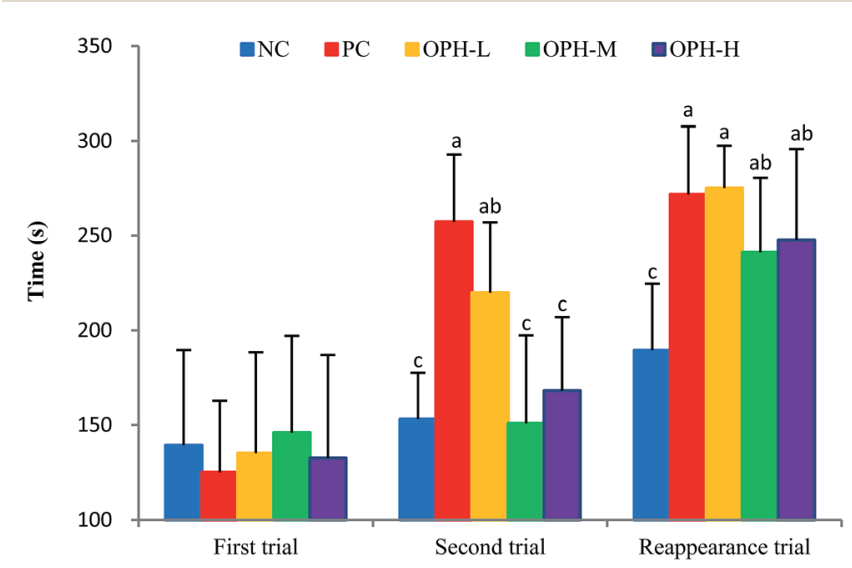

Fig. 3 Effect of OPH on step-through latency of dark/light avoidance test. The latency of five groups in the first trial showed no significant difference, and bar graphs followed no letters. In the second trial and reappearance trial, the same letters between the two groups meant no significant difference. Data were presented as means $\pm S D$. Bar graphs followed by different letters were significantly different $(P<0.05)$ according to variance analysis (ANOVA) using the SPSS software and means were compared by Duncan's multiple comparison post-test. Statistical differences were considered to be significant at $p<0.05$. the NC group $(P<0.05)$, while there was no significant difference between other OPH groups and the NC group. On the other hand, the level of GSH-Px was significantly increased in the $\mathrm{OPH}$ groups compared to the NC group $(P<0.05)$, which indicated that all the $\mathrm{OPH}$ groups could increase enzymatic antioxidant activity and reduce oxidative stress in the body. Additionally, MDA was one of the end products in the lipid metabolism, and increasing MDA levels reflected the damage to tissue and cell caused by accumulation of free radicals. ${ }^{45}$ As shown in Fig. 4, the MDA levels of all OPH groups were significantly lower than

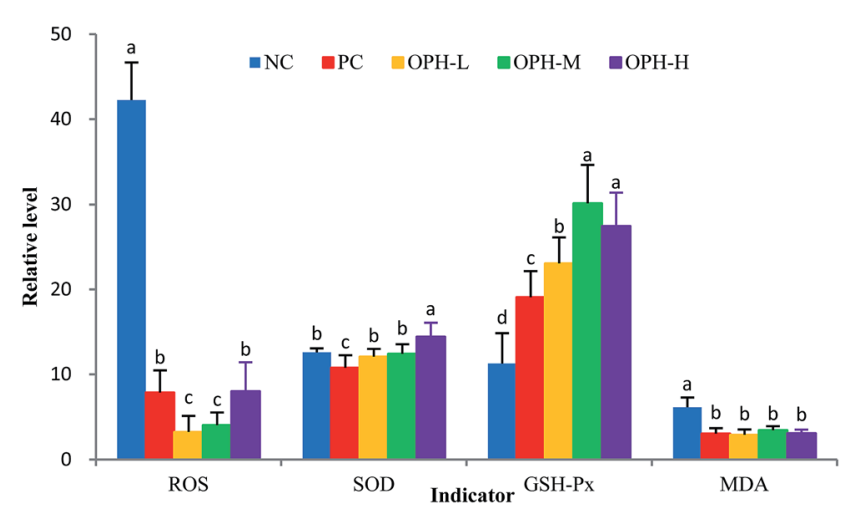

Fig. 4 Effect of OPH on indicators related to oxidative stress of mice. The vertical units of groups were $\%, \cup \mathrm{mg}^{-1}, \mathrm{U} \mathrm{mg}^{-1}$ and $\mathrm{mmol} \mathrm{mg}^{-1}$, respectively. Data were presented as means $\pm S D$. Bar graphs followed by different letters were significantly different $(P<0.05)$ according to variance analysis (ANOVA) using the SPSS software and means were compared by Duncan's multiple comparison post-test. Statistical differences were considered to be significant at $p<0.05$. 
that of the NC group; moreover, the MDA level of the PC group was also significantly lower than that of the NC group $(P<0.05)$. Therefore, the $\mathrm{OPH}$ groups could significantly relieve oxidative stress in vivo.

In addition, there are some studies illustrating that learning and memory ability is associated with oxidative stress. For example, Gao et al. $^{\mathbf{4 6}}$ found that fucoidan from brown algae could ameliorate $A \beta$-induced memory impairments in rats by increasing the homogenate levels of SOD and GSH-Px, and decreasing the level of MDA. Zhou et al. ${ }^{\mathbf{4 7}}$ exhibited that mussel oligopeptides could improve cognitive learning, memory ability and protect the hippocampal neurons. In addition, GSH, SOD and GSH-Px activities were increased and MDA level was significantly decreased in mice fed with mussel oligopeptides. It was found that antioxidant therapy prevented learning and memory deficits induced by $\mathrm{A} \beta$ in rats. Therefore, OPH had in vivo antioxidant activity, indicating that it might have the potential to improve learning and memory ability.

\subsection{Effect of OPH on indicators related to improving memory and inflammatory reaction}

3.8.1 AChE and ChAT. Biological studies have found that the central cholinergic system is closely linked to learning and memory, and that normal functioning of central cholinergic nerves is essential for learning and memory. ${ }^{48}$ AChE present in cholinergic axons and in some cholinoceptive pyramidal cortical neurons. A previous study reported that AChE activity is considered to be a potential therapeutic approach that can slow or mitigate the progression of $\mathrm{AD}^{49}$ Furthermore, ChAT is the major player of the cholinergic system, and is involved in acetylcholine synthesis and breakdown. ${ }^{50} \mathrm{~A}$ decrease in ChAT activity, as well as inflammatory response, was found in the hippocampus and cerebral cortex of AD patients. ${ }^{29}$ Additionally, AChE and ChAT may play a substantial part in the improvement of learning and memory ability. However, synthetic drugs have long-term safety problems and a negative consumer perception. ${ }^{\mathbf{8}}$ For these reasons, the demand for natural AChE inhibitors has recently increased. For example, Hirbod et al. ${ }^{51}$ have found the leaf and stem extractions of the Aquilaria were safe for use as natural AChE inhibitors, an alternative to berberine for the treatment of AD. In addition, Zhao et al. ${ }^{52}$ studied rhizome extractions from Anemarrhena asphodeloides Bge., which is widely used in traditional Chinese medicine, and the results indicated that rhizome extraction offered protection against scopolamine-induced deficits in learning and memory, possibly by inhibiting AChE and preventing oxidative stress damage.

In this section, we have measured the AChE activity and ChAT activity in the brain. Table 5 showed that the OPH-H group significantly inhibited AChE activity by $13.88 \mathrm{mM} \mathrm{min} \mathrm{m}^{-1}$ compared to that of the NC group of $28.89 \mathrm{mM} \mathrm{min}^{-1}(P<0.05)$. Interestingly, the OPH-L, OPH-M and PC groups did not decrease the AChE activity compared to that of the NC group.

Soodi et al. ${ }^{53}$ demonstrated that learning and memory deficit was associated with reduced ChAT in the hippocampus. As shown in Table 5, the expression of ChAT by 53.77 and 65.54 $\mathrm{mM} \min ^{-1}$ in the OPH-L and OPH-M were significantly increased in contrast to the NC group $(P<0.05)$. In addition, the PC group also increased the expression of ChAT compared to that of the NC group $(P<0.05)$. However, the OPH group showed no dose-dependence, and there was no statistical significance between the OPH-H and NC groups. As such, we assumed that there were great individual differences in animal experiments.

3.8.2 Inflammatory factors: TNF- $\alpha$, IL-6 and IL-1 $\beta$. As one of the most generic response in innate immunity system, inflammation is believed to play crucial roles in the pathogenesis of various diseases. ${ }^{54,55}$ During the dark/light avoidance test, the mouse carried out a lot of exercise and was frightened. There were some studies have shown that strenuous exercise or intense muscular work could induce oxidative stress and inflammation. ${ }^{43,56}$ Furthermore, when the body had an inflammatory response, the immune defense system could respond actively to inflammation by releasing pro-inflammatory cytokines, such as TNF- $\alpha$, IL- 6 and IL-1 $\beta$. However, high levels of these cytokines could cause systemic complications. ${ }^{57}$

Based on the above theories, we have investigated the effects of $\mathrm{OPH}$ on pro-inflammatory cytokines production, including TNF- $\alpha$, IL-6 and IL-1 $\beta$. The TNF- $\alpha$ was elevated in several neuropathological states that are associated with learning and memory deficits ${ }^{58}$ and could stimulate the production or expression of IL-6 and IL-1 $\beta .^{59}$ As shown in Table 5, after treatment with $\mathrm{OPH}$, the TNF- $\alpha$ level decreased significantly in $\mathrm{OPH}-\mathrm{M}$ and OPH-H groups compared to that of the NC group $(P$ $<0.05)$. The result was consistent with those reported by Lee et al. ${ }^{60}$ who found oyster shell extract significantly inhibited the production of IL-1 $\beta$, IL- 6 , and TNF- $\alpha$ in a dose-dependent manner. Recent studies suggest IL-6 may sit in a pivotal position and serve as an important catalyst because administration of IL-6 improved spatial alternation behavior ${ }^{61}$ and positively

Table 5 Effect of OPH on AChE, ChAT, IL-1 $\beta$, IL- 6 and TNF- $\alpha$ of mice ${ }^{a}$

\begin{tabular}{|c|c|c|c|c|c|}
\hline Group & $\operatorname{AChE}\left(\mathrm{mM} \mathrm{min}{ }^{-1}\right)$ & $\operatorname{ChAT}\left(\mathrm{mM} \mathrm{min}{ }^{-1}\right)$ & $\mathrm{IL}-1 \beta\left(\mathrm{pg} \mathrm{mL} \mathrm{L}^{-1}\right)$ & IL-6 $\left(\mathrm{pg} \mathrm{mL} \mathrm{mL}^{-1}\right)$ & $\mathrm{TNF}-\alpha\left(\mathrm{pg} \mathrm{mL}^{-1}\right)$ \\
\hline NC & $28.89 \pm 3.88^{\mathrm{a}}$ & $37.97 \pm 2.14^{\mathrm{d}}$ & $66.19 \pm 11.17^{\mathrm{c}}$ & $81.33 \pm 4.02^{\mathrm{b}}$ & $388.72 \pm 29.32^{\mathrm{b}}$ \\
\hline PC & $28.51 \pm 3.71^{\mathrm{a}}$ & $44.94 \pm 4.13^{c}$ & $84.64 \pm 2.89^{\mathrm{b}}$ & $127.74 \pm 6.21^{\mathrm{a}}$ & $475.90 \pm 20.76^{\mathrm{a}}$ \\
\hline OPH-M & $26.19 \pm 3.07^{\mathrm{a}}$ & $65.54 \pm 2.16^{\mathrm{a}}$ & $86.97 \pm 7.30^{\mathrm{b}}$ & $77.39 \pm 5.46^{\mathrm{c}}$ & $359.29 \pm 73.56^{\mathrm{c}}$ \\
\hline OPH-H & $13.88 \pm 3.82^{\mathrm{b}}$ & $38.68 \pm 2.18^{d}$ & $52.31 \pm 7.44^{\mathrm{d}}$ & $77.01 \pm 5.94^{c}$ & $338.05 \pm 13.71^{\mathrm{d}}$ \\
\hline
\end{tabular}

${ }^{a}$ Data were presented as means \pm SD. Values followed by different superscript in the same columns were significantly different according to variance analysis (ANOVA) using the SPSS software and means were compared by Duncan's multiple comparison post-test. Statistical differences were considered to be significant at $p<0.05$. 

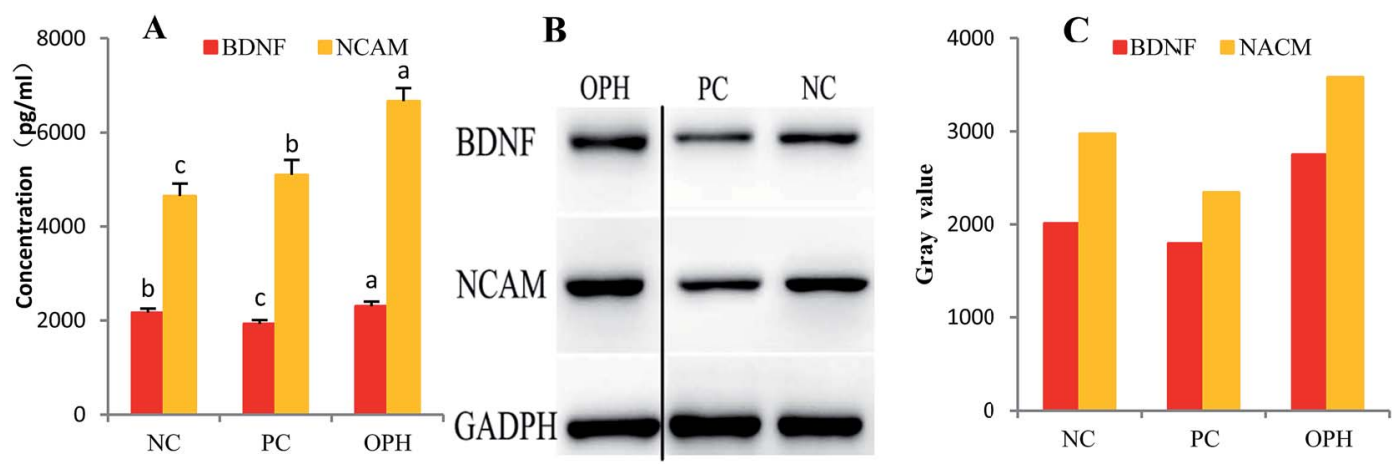

Fig. 5 Immunoblot analysis of BDNF and NCAM in the hippocampus of the OPH, PC and NC treated mice. (A) Quantitative analysis showed that the hippocampal levels of BDNF and NCAM of the OPH group were obviously higher than that of the NC group. (B) Western blot from the NC and PC groups showed weak hippocampal BDNF and NCAM compared with the OPH group. The OPH group data and NC and PC group's data were spliced together because there were other data between them in the initial gel. (C) Gray value of BDNF and NCAM in the hippocampus of mice treated with the OPH, PC and NC. Data were presented as means \pm SD. Bar graphs followed by different letters were significantly different $(P<$ 0.05 ) according to variance analysis (ANOVA) using the SPSS software and means were compared by Duncan's multiple comparison post-test. Statistical differences were considered to be significant at $p<0.05$.

correlated with deficits in cognition in humans. ${ }^{62}$ Therefore, we had also studied the level of IL-6 after treatment with OPH and the result showed that the IL-6 level decreased significantly in $\mathrm{OPH}-\mathrm{M}$ and $\mathrm{OPH}-\mathrm{H}$ groups compared to that of the NC group $(P$ $<0.05)$. Furthermore, it has been widely described that immune activation, such as IL-1 $\beta$ caused deficits in learning and memory. ${ }^{63}$ As shown in Table 5, IL-1 $\beta$ level decreased dramatically with OPH-H treatment compared to that of the NC group $(P$ $<0.05$ ). This result indicated that $\mathrm{OPH}$ could attenuate the production of pro-inflammatory cytokines, including TNF- $\alpha$, IL$1 \beta$, and IL-6. Whereas IL-1 $\beta$, IL- 6 and TNF- $\alpha$ levels in the PC group was increased dramatically compared to that of the NC group. Several types of drugs are used to treat inflammatory disorders, but they cause adverse side effects. We inferred that the PC group would demonstrate some inflammatory activity when compared to the normal mice, and that natural products are alternatives to these drugs for treatment.

3.8.3 BDNF and NCAM. BDNF is a critical molecule for learning and memory. ${ }^{64,65} \mathrm{In} \mathrm{AD}$, there is a significant downregulation of BDNF messenger RNA and protein, which is strongly implicated in loss of memory and cognitive function. ${ }^{66}$ As shown in Fig. 5A, quantitative analysis showed that hippocampal level of BDNF increased to $2302 \mathrm{pg} \mathrm{mL}^{-1}$ in the $\mathrm{OPH}$ group was obviously higher than that by $2167 \mathrm{pg} \mathrm{mL}^{-1}$ in the NC group $(P<0.05)$. However, the PC group was down-regulated of

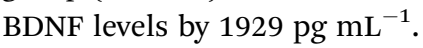

NCAM are complexes of transmembrane proteins critical for cell-cell interactions. Crucially, subsequent research demonstrated that NCAMs retain these functions in the mature adult brain with regards to cognitive and mimetics abilities. ${ }^{67}$ In this section, we have also investigated the NCAM levels and found that these levels in OPH group were significantly up-regulated compared to that of the NC group (Fig. 5A).

Furthermore, as shown in Fig. 5B, western blot from the PC group showed weak hippocampal BDNF and NCAM expressions compared with the NC group. These results reinforced that BDNF and NCAM expressions were observed in the OPH-treated groups. Additionally, the gray values of BDNF and NCAM in the hippocampus of the OPH group were higher than that of NC group in Fig. 5C. The result also showed that the OPH could improve learning and memory ability by up-regulating the BDNF and NCAM levels.

\section{Conclusions}

In this work, we confirmed the preparation conditions of $\mathrm{OPH}$ by RSM and proved that OPH had a spatial learning and memory capacity. In the Morris water maze test, the mice in OPH group showed shorter escape latency and total swimming distance; in the dark/light avoidance test, the mice in $\mathrm{OPH}$ group also showed longer step-through latency. Refer to other research works, we inferred that learning and memory ability was associated with oxidative stress and inflammatory reaction, and the related indexes confirmed our conjecture. However, the individual differences between mice were too great, and the OPH group did not show dose-dependent behavior in some experimental results, and, if possible, we would adjust the $\mathrm{OPH}$ dose in a future animal experiment. Furthermore, we also measured related indexes such as AChE and ChAT to proof the OPH could be a candidate functional food for the improvement of learning and memory. On the basis of the results obtained in this work, more work is worthy to be done to reveal the mechanisms on learning and memory ability of $\mathrm{OPH}$, and further purification and identification of $\mathrm{OPH}$ was needed.

\section{Conflicts of interest}

The authors declare that there is no conflict of interests regarding the publication of this paper.

\section{Acknowledgements}

The study was supported by the Key Research and Development Program of Shandong Province (2017YYSP018), National 
Natural Science Foundation of China (No. 41506175), Qingdao Science and Technology Project (No. 17-3-3-21-nsh), Science and Technology Development Plan Project of Shandong Province (2016YYSP010) and the Key Research Program of the Chinese Academy of Sciences (No. KFZD-SW-106).

\section{References}

1 L. Ma, L. Chang, X. Y. Chen and R. L. Zhou, PLoS One, 2017, 12, 1-19.

2 D. A. B. D. Boyd-Kimball, Brain Pathol., 2004, 14, 426-432.

3 C. Supnet and I. Bezprozvanny, Cell Calcium, 2011, 50, 303309.

4 M. S. Rafii and P. S. Aisen, BMC Med., 2015, 13, 1-7.

5 R. Brookmeyer, E. Johnson, K. Ziegler-Graham and H. M. Arrighi, Alzheimer's Dementia, 2007, 3, 186-191.

6 M. P. Mattson, Nature, 2004, 430, 631-639.

7 H. R. Amanatkar, B. Papagiannopoulos and G. T. Grossberg, Expert Rev. Neurother., 2017, 17, 7-16.

8 E. D. Roberson and L. Mucke, Science, 2006, 314, 781-784.

9 H.-J. Chai, C.-J. Wu, S.-H. Yang, T.-L. Li and B. Sun Pan, J. Funct. Foods, 2016, 24, 438-449.

10 J. A. Pillai, A. Bonner-Jackson, E. Walker, L. Mourany and J. L. Cummings, Dementia Geriatr. Cognit. Disord., 2014, 38, 224-233.

11 H. Chen, M. Zhao, L. Lin, J. Wang, D. Sun-Waterhouse, Y. Dong, M. Zhuang and G. Su, Food Res. Int., 2015, 78, 216-223.

12 H. J. Heo, S. J. Choi, S. G. Choi, D. H. Shin, J. M. Lee and C. Y. Lee, J. Food Sci., 2008, 73, H28-H32.

13 Z. Li, X. Chen, W. Lu, S. Zhang, X. Guan, Z. Li and D. Wang, Int. J. Mol. Sci., 2017, 18, 1-14.

14 C. S. Raine, Neurobiol. Aging, 2000, 21, 437-440.

15 S. Xiao, D. Zhou, P. Luan, B. Gu, L. Feng, S. Fan, W. Liao, W. Fang, L. Yang, E. Tao, R. Guo and J. Liu, Biomaterials, 2016, 106, 98-110.

16 S. Duan, X. Guan, R. Lin, X. Liu, Y. Yan, R. Lin, T. Zhang, X. Chen, J. Huang, X. Sun, Q. Li, S. Fang, J. Xu, Z. Yao and H. Gu, Neurobiol. Aging, 2015, 36, 1792-1807.

17 B. Bloem, R. B. Poorthuis and H. D. Mansvelder, Front. Neural Circuits, 2014, 8, 1-16.

18 M. H. Kim, S.-H. Kim and W. M. Yang, Planta Med., 2014, 80, 1249-1258.

19 Q. Wang, W. Li, Y. He, D. Ren, F. Kow, L. Song and X. Yu, Food Chem., 2014, 145, 991-996.

20 J. Wang, J. Hu, J. Cui, X. Bai, Y. Du, Y. Miyaguchi and B. Lin, Food Chem., 2008, 111, 302-308.

21 X. C. He, Y. Zhang, F. Yu and Z. N. Yu, Fish Shellfish Immunol., 2011, 31, 1247-1250.

22 Y. K. Wang, H. L. He, G. F. Wang, H. Wu, B. C. Zhou, X. L. Chen and Y. Z. Zhang, Mar. Drugs, 2010, 8, 255-268.

23 T. Xu, J. Xie, B. Zhu, X. Liu and X. Wu, PLoS One, 2014, 9, e95859.

24 H. S. Lin, W. H. Cao, H. Y. Lu, C. H. Zhang, X. M. Qin and M. Xie, Food Sci. Technol., 2013, 38, 37-41.

25 X. Q. Wang, R. E. Xing, S. Liu, H. H. Yu, K. C. Li, Z. Y. Chen and P. C. Li, Chin. J. Oceanol. Limnol., 2014, 33, 159-168.
26 A. Smeriglio, M. Denaro, D. Barreca, A. Calderaro, C. Bisignano, G. Ginestra, E. Bellocco and D. Trombetta, Int. J. Mol. Sci., 2017, 18, 1-13.

27 M. S. Blois, Nature, 1958, 181, 1199-1200.

28 M. Nishikimi, N. Appaji Rao and K. Yagi, Biochem. Biophys. Res. Commun., 1972, 46, 849-854.

29 C. L. Lee, T. F. Kuo, J. J. Wang and T. M. Pan, J. Neurosci. Res., 2007, 85, 3171-3182.

30 Y. Liu, Y. F. Xu, L. Zhang, L. Huang, P. Yu, H. Zhu, W. Deng and C. Qin, Int. J. Mol. Sci., 2017, 18, 1-17.

31 Y. Yamaguchi, H. Miyashita, H. Tsunekawa, A. Mouri, H.-C. Kim, K. Saito, T. Matsuno, S. Kawashima and T. Nabeshima, J. Pharmacol. Exp. Ther., 2006, 317, 10791087.

32 X. Fang, N. Xie, X. Chen, H. Yu and J. Chen, Food Bioprod. Process., 2012, 90, 676-682.

33 J.-Y. Ko, J.-H. Lee, K. Samarakoon, J.-S. Kim and Y.-J. Jeon, Food Chem. Toxicol., 2013, 52, 113-120.

34 J. P. Jia, Y. G. Zhou, J. Z. Lu, A. Y. Chen, Y. Z. Li and G. L. Zheng, J. Sci. Food Agric., 2010, 90, 635-640.

35 G. Zha, V. P. Chen, W. K. Luk, X. Zou, R. C. Choi and K. W. Tsim, Chem.-Biol. Interact., 2013, 203, 277-281.

36 Z. Y. Zhao, Q. Zhang, Y. F. Li, L. L. Dong and S. L. Liu, Carbohydr. Polym., 2015, 119, 101-109.

37 X. Q. Wang, R. Xing, Z. Y. Chen, H. H. Yu, R. F. Li and P. C. Li, Food Funct., 2014, 5, 2113-2119.

38 I. O. Ishola, F. M. Adamson and O. O. Adeyemi, Metab. Brain Dis., 2017, 32, 235-245.

39 L. Liu, J. Cao, J. Chen, X. Zhang, Z. Wu and H. Xiang, Neurosci. Lett., 2016, 631, 30-35.

40 Z. Mao, Y. L. Zheng and Y. Q. Zhang, Int. J. Mol. Sci., 2011, 12, 114-127.

41 H. E. Seifried, D. E. Anderson, E. I. Fisher and J. A. Milner, J. Nutr. Biochem., 2007, 18, 567-579.

42 G.-W. Oh, S.-C. Ko, S.-Y. Heo, N. Van-Tinh, G. Kim, C. H. Jang, W. S. Park, I.-W. Choi, Z.-J. Qian and W.-K. Jung, Process Biochem., 2015, 50, 1318-1326.

43 N. B. J. Vollaard, J. P. Shearman and C. E. Cooper, Sports Med., 2005, 35, 1045-1062.

44 C. Noschang, R. Krolow, D. M. Arcego, A. P. Toniazzo, A. P. Huffell and C. Dalmaz, Int. J. Dev. Neurosci., 2012, 30, 285-291.

45 M. E. Inal, G. Kanbak and E. Sunal, Clin. Chim. Acta, 2001, 305, 75-80.

46 Y. Gao, C. Li, J. Yin, J. Shen, H. Wang, Y. Wu and H. Jin, Environ. Toxicol. Pharmacol., 2012, 33, 304-311.

47 Y. Zhou, Y. Dong, Q. Xu, Y. He, S. Tian, S. Zhu, Y. Zhu and X. Dong, Food Chem. Toxicol., 2013, 59, 412-420.

48 Y.-s. Li, Y.-f. Hong, J. He, J.-x. Lin, Y.-l. Shan, D.-y. Fu, Z.-p. Chen, X.-r. Ren, Z.-h. Song and L. Tao, Biol. Pharm. Bull., 2013, 36, 764-771.

49 Y. Zhen and F. Jian, Curr. Alzheimer Res., 2004, 1, 241-248. 50 X. Hu, J. Wang, Q. Zhang, X. Duan, Z. Chen and Y. Zhang, J. Surg. Res., 2016, 206, 307-315.

51 B. Hirbod, M. Jamaludin, P. Mohammadjavad and A. R. Hussin, Curr. Alzheimer Res., 2014, 11, 206-214. 
52 X. Zhao, C. Liu, Y. Qi, L. Fang, J. Luo, K. Bi and Y. Jia, Metab. Brain Dis., 2016, 31, 1455-1461.

53 M. Soodi, S. Saeidnia, M. Sharifzadeh, H. Hajimehdipoor, A. Dashti, M. R. Sepand and S. Moradi, Metab. Brain Dis., 2016, 31, 395-404.

54 L. Ferrero-Miliani, O. H. Nielsen, P. S. Andersen and S. E. Girardin, Clin. Exp. Immunol., 2007, 147, 227-235.

55 R. C. Cheung, T. B. Ng, J. H. Wong, Y. Chen and W. Y. Chan, Appl. Microbiol. Biotechnol., 2016, 100, 1645-1666.

56 B. K. Pedersen, K. Ostrowski, T. Rohde and H. Bruunsgaard, Can. J. Physiol. Pharmacol., 1998, 76, 505-511.

57 L. Bosca, M. Zeini, P. G. Traves and S. Hortelano, Toxicology, 2005, 208, 249-258.

58 M. P. Butler, J. J. O'Connor and P. N. Moynagh, Neuroscience, 2004, 124, 319-326.

59 B. B. Aggarwal and K. Natarajan, Eur. Cytokine Network, 1996, 7, 93-124.
60 S.-Y. Lee, H.-J. Kim and J.-S. Han, Prev. Nutr. Food Sci., 2013, 18, 23-29.

61 N. L. Sparkman, J. B. Buchanan, J. R. R. Heyen, J. Chen, J. L. Beverly and R. W. Johnson, J. Neurosci., 2006, 26, 10709-10716.

62 J. D. Weaver, M. H. Huang, M. Albert, T. Harris, J. W. Rowe and T. E. Seeman, Neurology, 2002, 59, 371-378.

63 C. Cunningham and D. J. Sanderson, Brain, Behav., Immun., 2008, 22, 1117-1127.

64 M. Fahnestock, Future Neurol., 2011, 6, 627-639.

65 H. Hori, R. Yoshimura, A. Katsuki, K. Atake, R. Igata, Y. Konishi, H. Beppu and H. Tominaga, Int. J. Mol. Sci., 2017, 18, 1-8.

66 M. Baydyuk and B. J. Xu, Front. Neural Circuits, 2014, 8, 1-10. 67 G. Dallérac, C. Rampon and V. Doyère, Neurochem. Res., 2013, 38, 1163-1173. 